\title{
A DC Arc Model for Series Faults in Low Voltage Microgrids
}

\author{
F. M. Uriarte A. L. Gattozzi J. D. Herbst H. B. Estes \\ Senior Member, \\ T. J. Hotz A. Kwasinski, \\ R.E. Hebner \\ IEEE \\ Member, \\ IEEE \\ Fellow, IEEE
}

Center for Electromechanics

The University of Texas at Austin, USA

\begin{abstract}
This paper presents a dc arc fault model to simplify the study of a critical issue in dc microgrids: series faults. The model is derived from a hyperbolic approximation of observed arc voltage and current patterns, which permit analyzing the arc in terms of its resistance, power, energy, and quenching condition. Recent faults staged by the authors on a dc microgrid yielded enough fault data to develop an arc model. These faults were of three types: constant-gap speed, fixed-gap distance, and accelerated gap. The results in this paper compare experimental and simulation results for the three fault types. It is concluded that because the instantaneous voltage, current, power, and energy waveforms produced by the model agree well with experimental results, the model is suitable for transient simulations.
\end{abstract}

Index Terms - arc, computer, dc, energy, fault, flash, hazard, power, microgrid, model, series, simulation, transient.

\section{INTRODUCTION}

$\mathrm{T}$ HE potential for high operational flexibility, availability, reduced footprint, and better load compatibility of $\mathrm{dc}$ microgrids is of increasing interest to military bases, data centers, airplanes, and forthcoming all-electric ships. A major advantage of $\mathrm{dc}$ microgrids is their ease of integration to renewable energy sources and energy storage. Difficult arc fault interruption, however, has traditionally hindered mainstream application of dc microgrids. Understanding series faults in dc distribution systems can contribute to the development of new smart grid architectures both purely dc, as recently proposed by Korea Power Electric Company [1], and hybrid dc and ac, as suggested by the US DOE in its 20102014 multi-year plan [2].

Series faults occur at unintended points of discontinuity within an electrical circuit. These circuit imperfections often emerge as a contact separation that leads to an inline series-arc condition. The arc produced across the galvanic discontinuity is a fault where electrical current is maintained through the formation of a high-temperature plasma arc which ionizes the dielectric medium. . These faults may appear at arbitrary locations on a de microgrid and are particularly difficult to detect for very low values of series-arc resistance produce only minimal changes to system current and voltage levels.

This project was supported in part by the Office of Naval Research under grant \# N00014-08-1-0080 (e-mails: \{f.uriarte, a.gattozzi, j.herbst, h.estes, t.hotz, r.hebner\}@cem.utexas.edu, akwasins@mail.utexas.edu).
Models of electric arcs have progressed, essentially, along three lines of investigation:

a) Physics-based models, relying on the mathematical description of the physical processes in an electric arc. These models combine electromagnetic, fluid dynamic, and thermodynamic equations resulting in complex systems of differential equations [3, 4].

b) Equivalent circuit models, attempting to capture the arc effects on an electric circuit in terms of either the arc's voltage-current relationship or its apparent impedance [5, $6]$.

c) Heuristic models, attempting a general description of the arc based on experimental data, often combining a macroscopic mathematical model, like the one in the equivalent circuit, with additional ad hoc parameters or equations to improve correlation with observations $[7,8]$.

This paper offers a contribution to the description of an electric arc and, consequently, to the modeling of a series fault for transient simulations of dc microgrids, which is in line with the heuristic approach. Analysis of typical experimental voltage-current signatures of said fault types show that the arc behavior is strongly dependent on surrounding environmental conditions as well as electrical time constants. Since in practice a power system's time constants are unknown, a simple - yet practical — series fault model is developed to be independent of these constants.

\section{DC ARC FAult TESTBED}

The Center for Electromechanics (CEM) at the University of Texas at Austin (UT-CEM) operates a MW-level dc microgrid that can be configured at a number of power and voltage levels, and operated from the grid or in island mode. (More information on UT-CEM's de microgrid can be found in [9-11].)

Three types of series faults were investigated on this dc microgrid: constant-speed gap, fixed-distance gap, and accelerated gap. Constant-speed series faults represent conductors tearing longitudinally at a constant speed. This behavior is observed in conductors, well supported mechanically, that initiate an arc due to, for example, internal mechanical failure or malfunction of a connector. Fixed-gap faults are series faults where the electrodes remain stationary (dwell) after reaching a predetermined distance. (Most arc models in the literature assume this fault type.) Accelerated series faults represent the separation of two conductor sections 
at an accelerated rate. This fault type is the most challenging to model because the arc does not reach a steady state behavior. This situation reflects, for example, an energized cable that breaks and falls. Although the arc in accelerated faults extinguishes rather quickly, it is desirable to know whether the rate of change of voltage and current introduce malign transients in power systems.

A photograph of the series fault testbed is shown in the closed position in Fig. 1. Two electrodes maintain electrical continuity between the source and the load before faults initiate. When a fault initiates, the anode slides to the right to produce contact separation. This separation (or gap) distance is noted as $x_{g a p}$ and represents the distance measured from the cathode to the anode. (It is noted that $x_{\text {gap }}$ represents gap distance and not arc length.)

An additional distance $x_{\text {crit }}$ is noted below the anode in Fig. 1. Since $x_{\text {crit }}$ marks the boundary between the arc's burning and quenching stages, the ratio $q=x_{\text {gap }} / x_{\text {crit }}$ is defined to indicate that when $q=1$, the arc reaches the quenching, leading to its extinction. This distance is a synthetic description of the arc's collapse and is used herein to describe the arc randomness, quenching instant, and gap resistance.

\section{ARC FAULt SignATURES}

In the course of an ongoing investigation, UT-CEM recently staged 30 faults on its dc microgrid under different source and load conditions. The source and load networks were routinely changed to assess their effect on series faults. Despite changing test conditions, measurements from numerous faults show consistent voltage and current envelope behaviors.

The voltage and current trajectories of the tests were generalized into common patterns over the various source and load conditions. One experimental dataset is shown, for example, in Fig. 2. This dataset corresponds to a constant speed fault where the electrodes separated at $2.54 \mathrm{~mm} / \mathrm{s}(0.1$ $\mathrm{in} / \mathrm{s})$. The trace labeled $v_{\text {gap }}$ in Fig. 2 is the voltage across the testbed electrodes (measured from anode to cathode) as the electrodes separate. The amplitude of $v_{\text {gap }}$ is noted on the right-side axis in per unit (p.u.), where the peak dc voltage is 1 p.u. Likewise, the trace labeled $i_{\text {gap }}$ shows the current through the testbed electrodes as the electrodes separate, starting from a peak dc current of 1 p.u.

The following descriptions explain the key traits of series dc arc faults labeled as points $A$ through $H$ in Fig. 2.

Point A: This point indicates when the fault begins (at time $\left.t_{\text {fault }}\right)$. Before $A, v_{\text {gap }}$ is equal to the electrode voltage drop (approx. $0 \mathrm{~V}$ ) and $i_{\text {gap }}$ is equal to the load current $I_{\text {load }}$. When the fault is staged at $t_{\text {fault }} \approx 1.9 \mathrm{~s}$, the gap distance $x_{\text {gap }}$ increases from 0 to $0^{+}$: an infinitesimal gap distance greater than zero.

Point B: When the arc forms across the electrodes, a turnon voltage $a$ appears across the electrodes. The voltage $a$, which is independent of $x_{g a p}$ and $V_{d c}$, depends on the material composition of the electrodes themselves (not arc current or voltage across electrodes). As $x_{\text {gap }}$ continues to increase, $v_{\text {gap }}$ increases as noted between points $B$ and $C$. The gradient of this voltage rise is noted as $b \mathrm{~V} / \mathrm{mm}$. Similar to $a, b$ is also independent of $x_{g a p}$ and $V_{d c}$, and is nearly constant for a wide range of experimental source voltage levels.

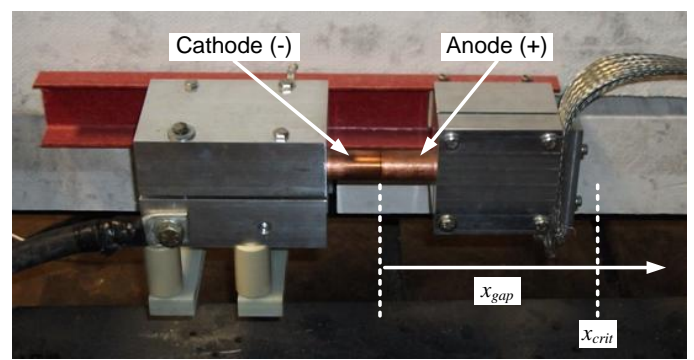

Fig. 1. Horizontal series fault, dc arc testbed at UT-CEM: two electrodes separate longitudinally to represent the separation of conductors.

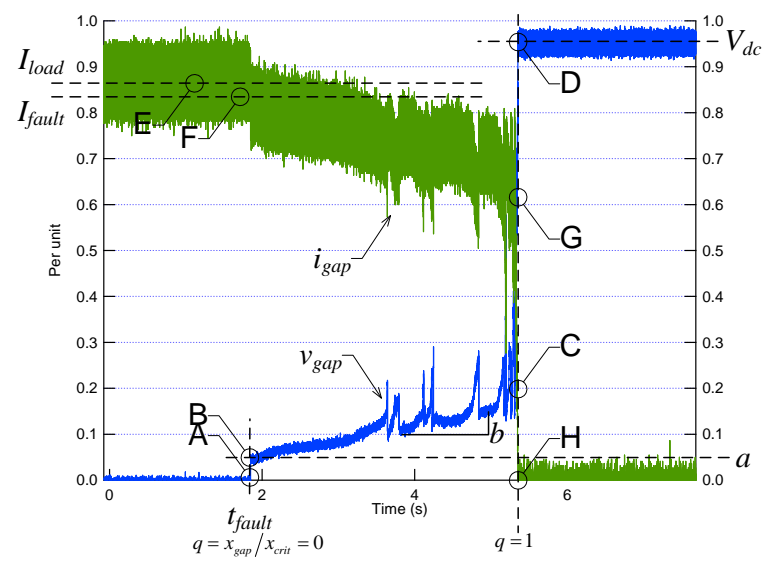

Fig. 2. Sample experimental data showing envelopes of gap voltage and current during a fault.

Point C: Several voltage spikes can be noted in $v_{g a p}$ between $B$ and $C$. These spikes represent unsuccessful quenching attempts. These attempts also appear in $i_{\text {gap }}$ as notches. (A successful quench would bring $v_{\text {gap }}$ to its opencircuit value of 1 p.u. and $i_{\text {gap }}$ to zero.) The near-instantaneous relationship noticed between $v_{\text {gap }}$ and $i_{\text {gap }}$ suggests that the arc impedance is resistive.

Point D: When $q=x_{\text {gap }} / x_{\text {crit }}>1$, the arc enters the quenching stage, which leads to its complete extinction at point $D$. This extinction is noticed by $v_{\text {gap }}$ rising abruptly from points $C$ to $D$ where $v_{\text {gap }}$ reaches the system voltage level.

Point E: The load current before the fault is $I_{\text {load }}$. When a fault initiates, the load current slightly reduces to $I_{\text {fault }}<I_{\text {load }}$, which make series faults appear as negligible changes in load current and difficult to detect. (The same occurs in highimpedance faults [12].)

Point F: The amount by which $i_{\text {gap }}$ decreases from $I_{\text {load }}$ to $I_{\text {fault }}$ is related to $a$, the electromotive force (EMF) impressed by the arc at point $B$. As $v_{\text {gap }}$ increases from $B$ to $C$ with a gradient of $b, i_{\text {gap }}$ decreases with a related gradient $f(b)$ from points $F$ to $G$.

Point $G$ : After $i_{\text {gap }}$ reaches $G$ at approximately $I_{\text {load }} / 2$, it decreases abruptly to 0 while $v_{\text {gap }}$ rises from point $C$ to $D$. 
Point H: At this point, $i_{g a p}=0$ and the arc extinguishes. The electrodes become an open circuit and the load is effectively disconnected from the power source.

\section{MODEL DERIVATION}

The previous section presented common traits of series dc arc faults. This section derives an arc model from a hyperbolic approximation of the arc's dynamic component of voltage and current. The resulting arc model is simple in implementation, does not require hard-to-find constants, is suitable for transient simulations, and is validated later in this paper.

Since the faults staged at UT-CEM took place at different dc voltage and current levels (up to $800 \mathrm{~V}$ and up to $200 \mathrm{~A}$ ), to simplify the analyses, the per-unit (p.u.) system is used here. The definitions of the base values are given in Table I.

Referring to Fig. 2, the arc's voltage and current can be decomposed into a voltage $v_{g a p} \approx v_{q}+e_{\text {gap }}$ and a current $i_{g a p} \approx$ $i_{q}-j_{g a p}$ as shown in Fig. 3. The details of these decompositions are given next.

\section{A. Gap Voltage}

Referring to Fig. 3, $v_{g a p}$ can be decomposed into a nonlinear hyperbolic-tangent function voltage $v_{q}$ and an electromotive force (EMF) pulse term $e_{\text {gap }}$ that is non-zero for $0<q<1$. This decomposition is given by $v_{g a p}=v_{q}+e_{\text {gap }}$. The trace for $v_{q}$ is shown in Fig. 3 and is approximated with the hyperbolictangent function

$$
v_{q}=V_{d c}\left(\frac{1}{2}+\frac{1}{2} \tanh (\alpha(q-1))\right)=V_{d c}\left(\frac{e^{2 q \alpha}}{e^{2 \alpha}+e^{2 q \alpha}}\right)
$$

where $V_{d c}$ is the average dc voltage before the fault (equal to $V_{\text {base }}$ in absence of rectification ripple), $\alpha$ is a variable that controls the slope of $v_{q}$, and $q=x_{\text {crit }} / x_{\text {gap }}$ was defined earlier. The pulse $e_{g a p}$ can be approximated by a nearly rectangular pulse of amplitude $a+b x_{g a p}$ as

$$
e_{g a p}=\frac{1}{2}\left(a+b x_{g a p}\right)(\tanh (\lambda q)-\tanh (\lambda(q-1))) .
$$

As shown in Fig. 3, $e_{g a p}$ is nonzero for $0<q<1$, and zero otherwise. In (2), $\lambda$ controls the rate at which $e_{g a p}$ rises and decays. A value that works well is $\lambda=100$, but others may be used as well. It is noted that $e_{g a p}$ dominates initially and $v_{q}$ dominates as the arc attempts to collapse. (The gap voltage $v_{g a p}=v_{q}+e_{g a p}$ is shown in Fig. 3 as a dotted line.)

\section{B. Gap Current}

Similar to the gap voltage, the gap current $i_{g a p}$ can also be decomposed into a nonlinear hyperbolic-tangent function $i_{q}$ and the system's response to the step-like $e_{g a p}$ noted previously. This decomposition is given by $i_{g a p}=i_{q}-j_{g a p}$.

The trace for $i_{q}$ shown in Fig. 3 and is approximated with the hyperbolic-tangent function

$$
i_{q}=I_{\text {load }}\left(\frac{1}{2}-\frac{1}{2} \tanh (\alpha(q-1))\right)=I_{\text {load }}\left(\frac{1}{1+e^{2 \alpha(q-1)}}\right)
$$

where $I_{\text {load }}$ is the average dc current before the fault (equal to $I_{\text {base }}$ in absence of rectification ripple). The term $j_{g a p}$ is the system's response to $e_{g a p}$ and can be approximated by

$$
j_{\text {gap }}=e_{\text {gap }} /\left(R_{\text {gap }}+R_{\text {load }}+R_{g}\right) \approx e_{\text {gap }} I_{\text {load }} / V_{d c} .
$$

In (4), $R_{g a p}$ is the gap resistance which is negligible in the region $0<q<1 ; R_{g}$ is the system's internal resistance which is small when compared to $R_{\text {load }}$. The dominant resistance in the denominator of (4) is $R_{\text {load }}$. More convenient than specifying the load resistance is to substitute $R_{\text {load }}=V_{d c} I_{\text {load }}$, which values are observed before the fault initiates.

TABLE I

DEFINITION OF BASE QUANTITIES USED IN THE PER UNIT SYSTEM

\begin{tabular}{ll}
\hline$V_{\text {base }}$ & $\begin{array}{l}\text { base voltage level (dc volts) equal to the peak dc } \\
\text { voltage measured at the output of the rectifier } \\
\text { terminals }\end{array}$ \\
\hline$I_{\text {base }}$ & $\begin{array}{l}\text { base current level (dc amps) equal to the peak dc } \\
\text { current measured at the rectifier output }\end{array}$ \\
\hline$P_{\text {base }}$ & base power level (W) equal to $V_{\text {base }} I_{\text {base }}$ \\
\hline$W_{\text {base }}$ & base energy level (Joules) equal to $P_{\text {base }} t_{\text {dur }}$ \\
\hline$t_{\text {dur }}$ & $\begin{array}{l}\text { fault duration in seconds (experimentally } \\
\text { determined) }\end{array}$ \\
\hline
\end{tabular}

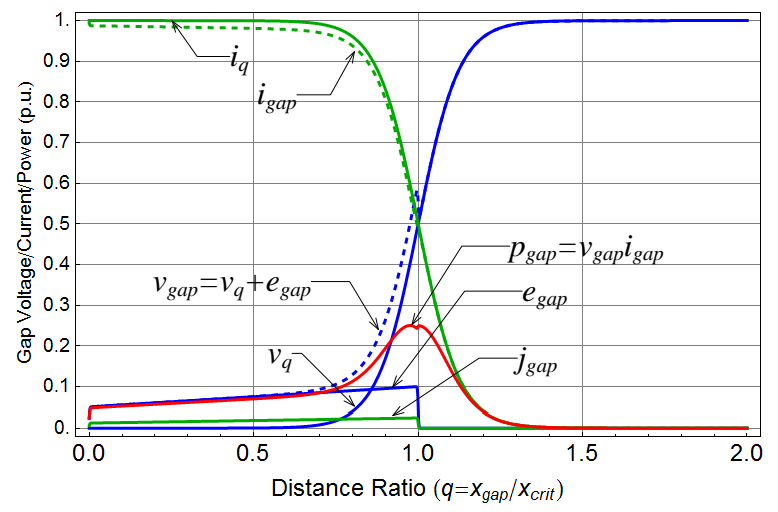

Fig. 3. Hyperbolic approximation of gap voltage and current trajectories. The arc power is shown as $p_{\text {gap }}$.

\section{Gap Resistance}

The simultaneous changes in experimental arc voltage and current show that the arc impedance is resistive. Although the quotient $R_{\text {gap }}=v_{q} / i_{g a p}$ approximates the gap resistance rather well, it produces a unnecessarily complicated and long expression. It was found that the influence of $j_{g a p}$ in $R_{g a p}$ is negligible, and can be removed in favor of a much simpler expression for $R_{g a p}$ without significant loss of accuracy. The simplified expression for $R_{\text {gap }}$ is given in (5).

$$
R_{\text {gap }}=\frac{v_{q}}{i_{\text {gap }}}=\frac{v_{q}}{i_{q}-j_{\text {gap }}} \approx \frac{V_{d c}}{I_{\text {load }}} e^{2 \alpha(q-1)} \Omega .
$$

The resistance in (5) requires specifying a value for $\alpha$. This value can be determined by assuming a value $R_{\text {closed }}$ when the electrodes are closed (at $q=0$ ) and solving for $\alpha$ in (6)-(7). 


$$
\begin{gathered}
\left.R_{\text {gap }}\right|_{q=0}=\frac{V_{d c}}{I_{\text {load }}} e^{-2 \alpha}=R_{\text {closed }} \Omega \\
\alpha=\frac{-1}{2} \ln \left(R_{\text {closed }} I_{\text {load }} / V_{d c}\right)
\end{gathered}
$$

For example, if $V_{d c} / I_{\text {load }}=800 \mathrm{~V} / 200 \mathrm{~A}$ and $R_{\text {closed }}=$ $0.001 \Omega$ (a typical breaker resistance in simulation programs), then $\alpha=4.14702$. The effect $\alpha$ has on $R_{\text {gap }}$ is shown in logarithmic scale in Fig. 4. Small $\alpha$ values (e.g., <2) result in high resistance when the electrodes are closed. Large values of $\alpha$ result in high $d R_{\text {gap }} / d t$ ratios that lead to spurious transients under inductive load. Using (7) to estimate $\alpha$ seems to give good results.

\section{Branch Model}

Using $R_{\text {gap }}$ in (5) and $e_{\text {gap }}$ in (2), an equivalent branch model can be formed, as shown on the left in Fig. 5. This branch is the proposed arc model of a dc series fault, and consists of a nonlinear resistance in series with an EMF source. (Shown on the right of Fig. 5 is the relative position of the branch model relative to arbitrary electrodes.) Referring to the left of Fig. 5, the current immediately after the fault is initiated is slightly less than the load current by the amount $j_{\text {gap }}$ due to the voltage drop $e_{\text {gap }}$, which is usually small in comparison to $I_{\text {load }}$. The slight reduction in load currents makes series faults difficult to detect initially.

\section{POWER ANALYSIS}

It is desirable to estimate the power losses of series dc arc faults. This section presents an analysis of the arc's power which is validated experimentally later.

\section{A. Arc Power}

The instantaneous power consumed by an arc fault is

$$
p_{\text {gap }}=v_{\text {gap }} i_{\text {gap }}=\left(v_{q}+e_{\text {gap }}\right) i_{\text {gap }}=v_{q_{\text {gap }}} i_{p_{R}}+\underbrace{e_{\text {gap }} i_{\text {gap }}}_{p_{E M F}} .
$$

Referring to the branch model on the left in Fig. 5, (8) is the sum of the power contributions of $R_{\text {gap }}$ and $e_{\text {gap }}$. Expanding (8) results in (9) and (10), which are the analytical approximations of the arc power.

$$
\begin{gathered}
p_{R}=\frac{1}{4} I_{\text {load }} f_{R}(q)(\tanh (\alpha(q-1))-1) \\
f_{R}(q)=\left(a+b x_{\text {gap }}\right)(\tanh (\lambda(1-q))+\tanh (\lambda q))+V_{d c}(\tanh (\alpha(q-1))-1) \\
p_{E M F}=\frac{1}{4} \frac{I_{\text {load }}}{V_{d c}}\left(a+b x_{\text {gap }}\right)(\tanh (\lambda-\lambda q)+\tanh (\lambda q)) f_{e}(q)
\end{gathered}
$$$$
f_{e}(q)=\left(\left(a+b x_{\text {gap }}\right)(\tanh (\lambda-\lambda q)+\tanh (\lambda q))+V_{d c}(\tanh ((q-1) \alpha)-1)\right)
$$

\section{B. Maximum Power Transfer}

Traces of (9) and (10) are shown in Fig. 6, assuming a resistive load for the circuit on the right of Fig. 5. It is noted that $p_{\text {gap }}$ peaks at $25 \%$ of the maximum available power ( $V_{\text {base }} I_{\text {base }}$ ), which corresponds to the one quarter coefficients in
(9) and (10). This peak value suggests that the arc begins to quench when $v_{\text {gap }}$ is half of the system dc voltage and $i_{\text {gap }}$ half of the nominal load current.

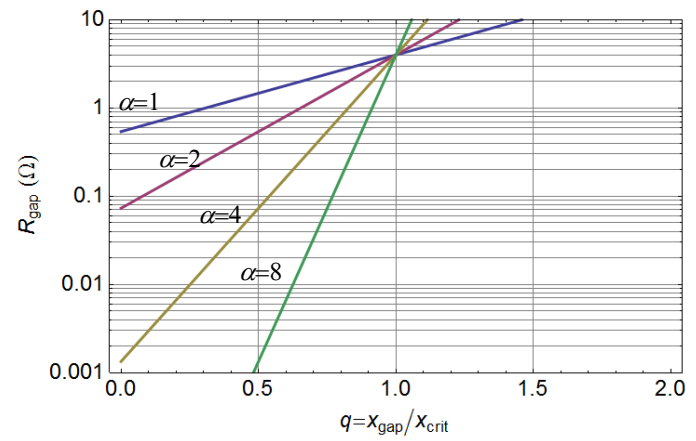

Fig. 4. Impact of $\alpha$ on $R_{\text {gap }}$ for $I_{\text {load }}=200 \mathrm{~A}$ and $V_{d c}=800 \mathrm{~V}$. To prevent significant voltage drops, $R_{g a p}$ should be negligible before a fault $(q \approx 0)$.

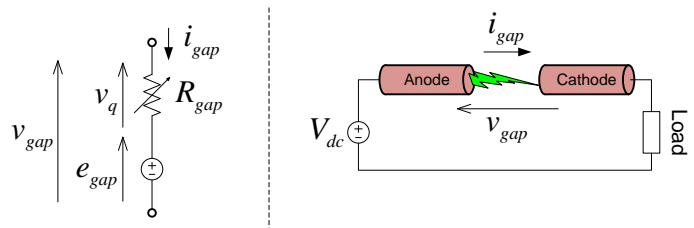

Fig. 5. Left: arc branch model showing voltage and current terms. Right: how the arc branch model relates to the separating electrodes.

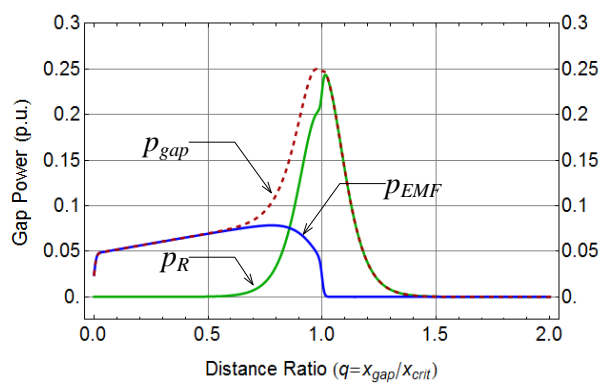

Fig. 6. Theoretical power consumed by arc resistance $\left(p_{R}\right)$, gap EMF $\left(p_{E M F}\right)$, and in total $\left(p_{\text {gap }}\right)$.

At this time, the arc absorbs half of the system voltage and carries half of the system current, which is one quarter of the pre-fault through power [13].

It should be noted that this is an observation about the approximate value of the transient power that can be dissipated in the arc. Arc cooling is a complex physical and chemical process so there is no a priori prediction that the arc would extinguish at approximately half of the system voltage and nominal current. From the data, however, it appears that the cooling rate is such that this is roughly true. The data were all taken with horizontal arcs in atmospheric air. Changes in these physical conditions would be expected to influence the point at which the arc cools and extinguishes.

\section{Reactive Systems}

In a resistive system, the peak power does not exceed 0.25 p.u. When energy-storage (i.e., reactive) elements are present in a system, the arc may draw stored energy and, thus, temporarily exceed the $p_{g a p}=0.25$ p.u. anticipated peak and even shift the peak power in time. 
Fig. 7 shows $p_{\text {gap }}$ as a dc system becomes more inductive. This peak shifting can occur, for example, when $p_{\text {gap }}=v_{\text {gap }} i_{\text {gap }}$ $=(0.4)(0.7)=0.28$ p.u., as observed in practice.

\section{ARC RANDOMNESS}

As the arc burns, non-intermittent fluctuations are observed in $v_{g a p}$ and $i_{g a p}$ in the experimental data (e.g., notice the variations in $v_{g a p}$ in Fig. 2.) These fluctuations are unsuccessful quenching attempts and modeled by randomizing the distance ratio $q=x_{\text {gap }} / x_{\text {crit }}$.

Consider the proposed branch model of Fig. 5 overlaid on the arc photograph in Fig. 8. The distance $x_{g a p}$ is a measurable distance; the critical distance $x_{\text {crit }}$ is not, and changes at each instant. It stands to reason that to observe the varying fluctuations in voltage and current, the series gap resistance $R_{\text {gap }}$ should behave randomly much like $x_{\text {crit }}$ does. Although $x_{\text {crit }}$ can be randomized, the exponent in $R_{g a p}$ of value $q$ suggests that it is more convenient to vary $q$ to produce rapid changes in $R_{\text {gap }}$. These rapid changes lead to the voltage and current fluctuations observed in the experimental data (e.g., Fig. 2).

A random function to jitter $q$ is given by

$$
q^{k+1}=q^{k}+\operatorname{rand}()^{10}
$$

where $k+1$ represents the current simulation time step, and $k$ the previous simulation time step. The random function for $q$ has a saw-tooth form that rises by random amounts each time $q$ is calculated. The exponent of 10 reduces the rate at which $q$ increases and is important to produce the notches observed experimentally in $v_{\text {gap }}$. In fixed gap arcs, to prevent $q$ from reaching unity, $q$ is reset to a new random value when $q>0.9$, which produces an arc that burns permanently, corresponding to experimental observations. On the contrary, if quenching is required in the mode (e.g., gaps opening at a constant speed), $q$ is allowed to surpass unity.

Accelerated faults were staged to replicate the case of fallen conductors. In this case, jittering was not observed. The rate at which the electrodes separate does not allow the arc to reach a steady state where the fluctuations are observed. If both $x_{g a p}$ and $x_{\text {crit }}$ are accelerated together such that when $q=1$ the simulation time equals the fault duration time $\left(t=t_{\text {dur }}\right)$, then $q$ can be calculated from (11), 9,800 the acceleration of gravity in $\mathrm{mm} / \mathrm{s}^{2}$, and $t^{k+1}$ the simulation time at time step $k+1$.

It must be noted that the use of random functions in the description of arc behavior is not new.

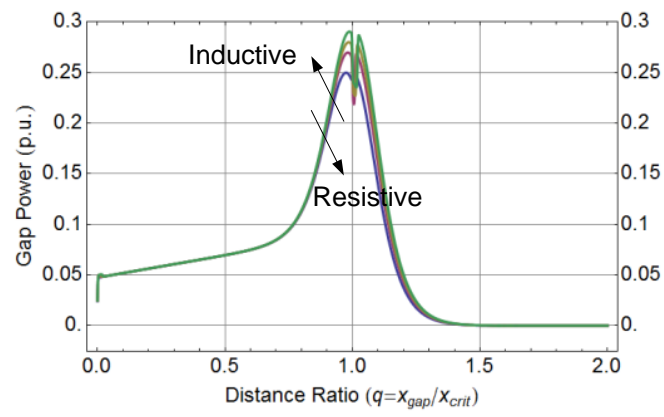

Fig. 7. Effect of system inductance on peak arc power.

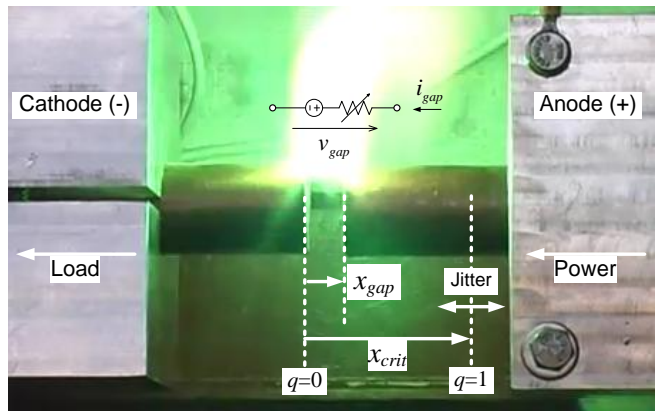

Fig. 8. UT-CEM's testbed during an arc fault. The proposed model is shown in relation to the electrodes. The ratio $q=x_{g a p} / x_{\text {crit }}$ jitters randomly between 0 and 1 to produce a time-varying resistance $R_{\text {gap }}$.

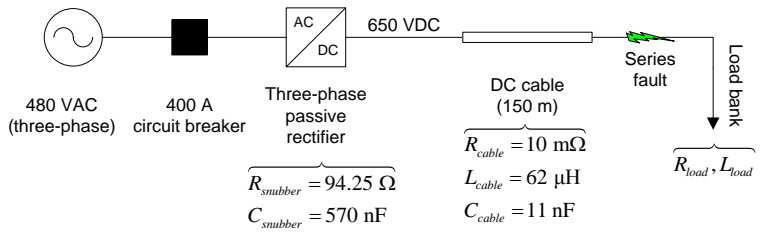

Fig. 9. Configuration of CEM's de microgrid for the fault studies [9, 10].

$q=x_{\text {gap }} / x_{\text {crit }}=\frac{1}{2} 9800 t^{2} / \frac{1}{2} 9800 t_{\text {dur }}^{2}=1 \Rightarrow q^{k+1}=\left(t^{k+1} / t_{\text {dur }}\right)^{2}$

For example, [14] uses a zero-mean Gaussian noise function to describe arc voltage fluctuations and a twoparameter non-deterministic function to describe arc elongation. This method has a long pedigree going back at least to [15].

\section{CASE STUdies}

This section compares experimental and simulation results for three fault types using the proposed model: constant speed, fixed distance, and accelerated. The $\mathrm{dc}$ migrogrid configuration used in all cases is shown in Fig. 9, where the annotated power apparatus parameters were obtained from data sheets or measured in the lab. The fault and other power system parameters for each case are listed in Table II.

\section{A. Case Study 1: Constant Speed Fault}

This case compares experimental and simulation results for a constant speed fault occurring as $x_{g a p}$ increases at the rate of $2.54 \mathrm{~mm} / \mathrm{s}(0.1 \mathrm{in} / \mathrm{s})$. 
TABLE II

SIMULATION AND FAULT PARAMETERS

\begin{tabular}{|c|c|c|}
\hline \multicolumn{3}{|c|}{ Common to all fault types } \\
\hline Description & Symbol & Value \\
\hline Gap EMF & $a$ & 0.01 p.u. \\
\hline EMF slope & $b$ & 0.005 p.u. \\
\hline$v_{q}$ slope & $\alpha$ & 4 \\
\hline$e_{\text {gap }}$ slope & $\lambda$ & 100 \\
\hline$V_{\text {base }}, I_{\text {base }}, P_{\text {base }}, W_{\text {base }}$ & & Noted on results \\
\hline Random number & $r$ & between 0 and 1 \\
\hline \multicolumn{3}{|c|}{ Constant Speed and Fixed Distance Faults } \\
\hline Gap separation rate & & $2.54 \mathrm{~mm} / \mathrm{s}$ \\
\hline Random function & $q$ & Equation (11) \\
\hline \multicolumn{3}{|c|}{ Load impedance: $R_{\text {load }}=3.986 \Omega, L_{\text {load }}=6.55 \mathrm{mH}, \tau=1.62 \mathrm{~ms}$} \\
\hline \multicolumn{3}{|c|}{ Accelerated Fault } \\
\hline Gap separation rate & & $1 / 29,800 t^{2} \mathrm{~mm} / \mathrm{s}$ \\
\hline Random function & $q$ & Equation (12) \\
\hline Load impedance: $R_{\text {load }}$ & $9 \Omega, L_{l o c}$ & $56 \mathrm{mH}, \tau=0.085 \mathrm{~ms}$ \\
\hline
\end{tabular}

This incremental separation permits observing arc behavior over a continuum of gap distances, which is important for assessing arc behavior as a function of gap distance.

Fig. 10 shows the experimental (left) and simulation results (right) side-by-side in p.u. The traces on the top row compare instantaneous $v_{\text {gap }}$ and $i_{\text {gap }}$, and the bottom row compares instantaneous power and cumulative energy. A comparison of the arc power and energy are important validation criteria to ensure the arc is modeled properly.

Also shown on the top row, as an annotation, are the base voltage $\left(V_{\text {base }}\right)$, base current $\left(I_{\text {base }}\right)$, fault duration $\left(t_{\text {dur }}\right)$, and experimental and simulation sampling rate $(d t)$. The bottom row also shows, with an arrowed-annotation, the peak power consumption $\left(P_{\text {gap }}\right)$, time at which this peak occurs $\left(t_{\text {peak }}\right)$, and the base power $\left(P_{\text {base }}\right)$ and energy levels $\left(W_{\text {base }}\right)$. From the top row, it is seen that $v_{\text {gap }}$ generally follows the experimental results.

Repeatable results cannot be obtained-not even in practice. For example, when the same fault was staged twice, the instantaneous voltages differed. This random behavior is included in the model through randomness in $R_{\text {gap }}$. The gap current $i_{\text {gap }}$ also approximates what is observed experimentally. In general, the simulated $v_{\text {gap }}$ and $i_{\text {gap }}$ are consistent with experimental results.

The bottom row in Fig. 10 shows the arc's instantaneous power and energy. The experimental peak power was slightly higher than the simulated results. This momentary difference, however, is short lived and not sustained. The arc energy was computed as the integral of $p_{\text {gap }}$ over the total experimental time. Both the arc power and energy agree reasonably well with experimental results and serve as validation metrics.

\section{B. Case Study 2: Fixed Distance Fault}

This case shows the arc behavior when the gap distance dwelled at $6.35 \mathrm{~mm}$ (0.25 in.). Consistent with the measurement instrumentation's storage capacity, the sampling period $d t$ was adjusted from $50 \mu$ s to $100 \mu$ s to capture longerburning arcs. Fig. 11 shows experimental and simulation results in the same order as Fig. 10. The simulated $v_{\text {gap }}$ and $i_{g a p}$ follow the trends of their experimental counterparts.

The experimental power shows a peak of 0.25 p.u., whereas the simulated peak was 0.21 p.u. These results are consistent with those predicted by Fig. 6, where the peak power is not expected to exceed 0.25 p.u. At times, and depending on whether there is parasitic (or intentional) energy storage in the system, this peak can be violated; yet, in this particular case, it was not.

The energy level of the experimental results shown in Fig. 11 reached 0.92 p.u. This value was exceeded by the simulation results, reaching 1 p.u. However, other simulations showed $W_{g a p}=0.96$ and $W_{g a p}=0.91$. This random arc behavior in the simulations mimics the one also observed in practice. Exact results cannot be replicated in the lab-not even with sequential experimental runs. Some of the discrepancies between a specific simulation and experimental results can be explained on the basis of the purposefully injected randomness.

\section{Case Study 3: Accelerated Fault}

The results for the accelerated faults are shown in Fig. 12. In accelerated faults the electrodes are accelerated apart at an acceleration of $9,800 \mathrm{~mm} / \mathrm{s}^{2}$. This fast separation of electrodes does not permit the arc to dwell at a steady-state. The experimental data show that the arcs quench approximately upon reaching the maximum power transfer point, and that accelerated faults only consumed between 0.02-0.05 p.u. (2$5 \%)$ of $W_{\text {base }}$. In all cases of accelerated experiments and simulations, the arc power was bound to $p_{\text {gap }}<0.25 \mathrm{p}$.u as shown by the peak power instant $t_{\text {peak }}$. Comparing experimental and simulated results, the voltage, current, power, and energy are consistent with the experimental results: both results show a total arc consumption of $W_{g a p}=0.021$ p.u.

\section{SUMMARY AND CONCLUSIONS}

An arc model for studying series faults in low-voltage dc microgrids was presented. The model does not require knowledge of physical (hard-to-get) time constants and can be implemented with a time-varying resistance. Because the instantaneous voltage and current behavior of the arc model is consistent with experimental results, the proposed model is suitable for electromagnetic transient simulations of series dc arc faults in low-voltage de microgrids.

Although no two data sets are identical, even under nominally the same environmental and electrical conditions, there is an underlying common behavior. While the exact magnitude and position of the individual spikes appear to be random, there is a consistent decrease in the gap current and increase in the gap voltage with time. The other apparently random feature is the gap spacing $x_{c r i t}$ at which the arc initiates rapid extinction. 

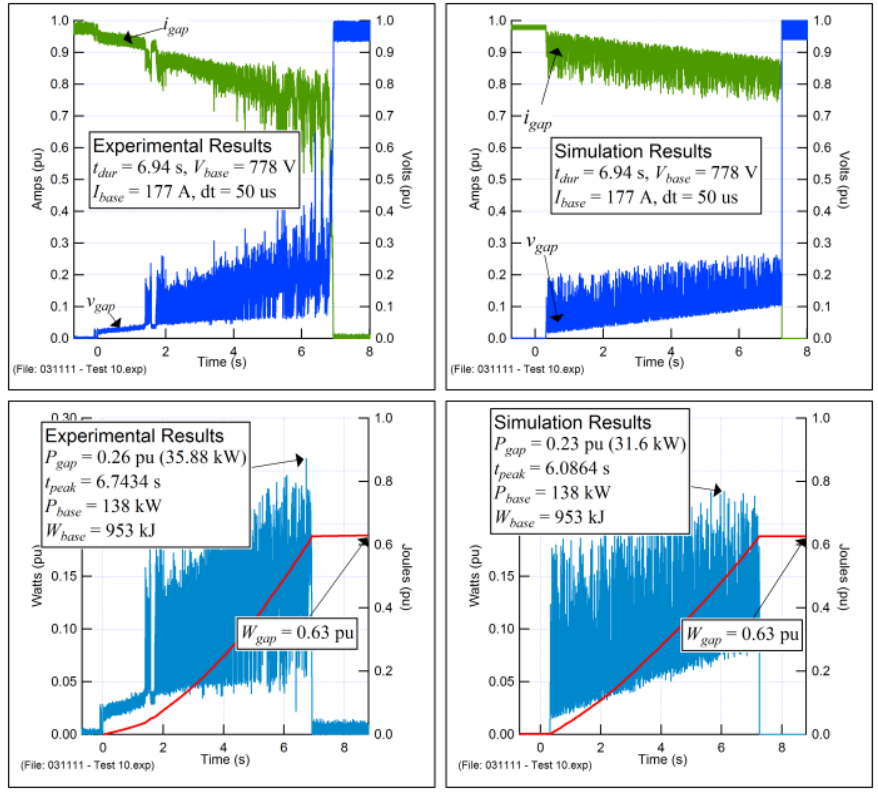

Fig. 10. Case study 1: constant speed fault (top row: instantaneous voltage and current; bottom row: instantaneous power and cumulative energy).

While modeling dc arc dynamics from first principles is making significant advances in fidelity [16], the modeling is still challenging. The reason for the challenge is that the arc is “... three-dimensional, unsteady, highly nonlinear, with large gradients, requiring a wide range of time and spatial scales for its description. In addition, chemical and thermodynamic nonequilibrium effects have to be considered ..." [16]. Consequently, the fundamental characterization of dc arcs has not yet advanced so that the arc dynamics in practical situations can be predicted.

At the same time, there is a increasing need to model dc distribution systems as they are being adopted in a growing number of situations in an attempt to improve efficiency [17, 18]. A critical unique feature of dc distribution is the selfsustaining series fault. The focus in this work is to provide reasonable models of that fault type that can be used in performance simulations. Consequently, the experimental data were fitted to analytical expressions. The specific analytical expressions used were suggested primarily by the shape of the data rather than by the physics and chemistry of the underlying processes. As the modeling of arcs themselves becomes more sophisticated, the analytical expressions for the arc behavior in a circuit can be refined.

It is not trivial to choose a random function for $q$, but one must be chosen. The criteria to do so must allow the arc to exist for an extended amount of time as observed experimentally, but also be suitable for the three fault types studied. Although the random functions presented here are suitable for the three fault types presented, they can be improved by tailoring them to each fault type if additional fidelity is required.
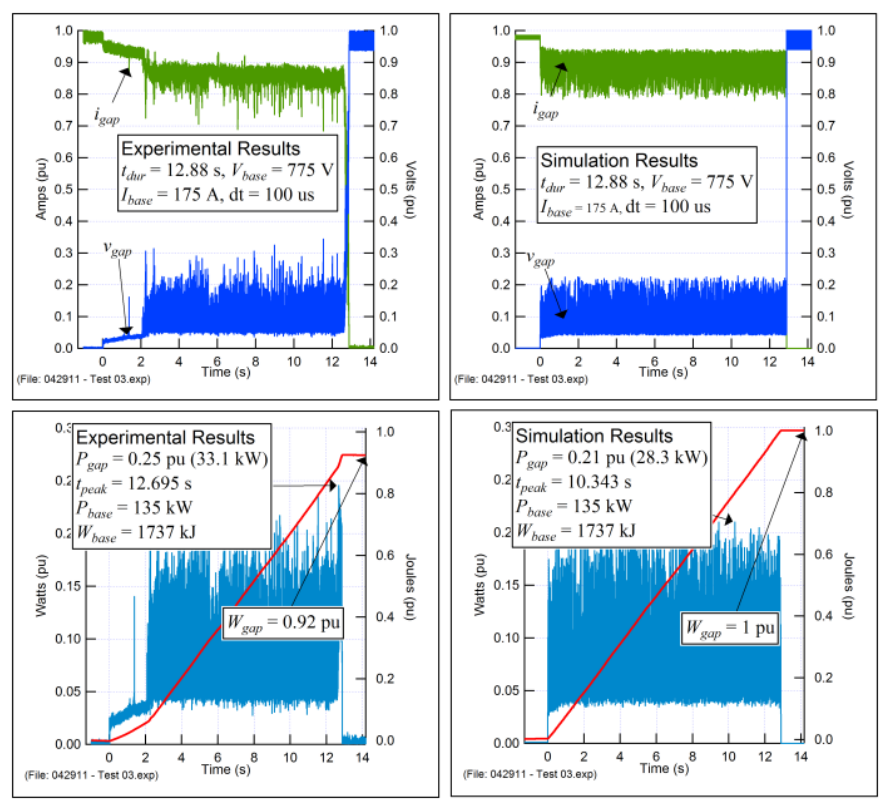

Fig. 11. Case study 2: gap opened to a fixed gap-distance fault (top row: instantaneous voltage and current; bottom row: instantaneous power and cumulative energy).
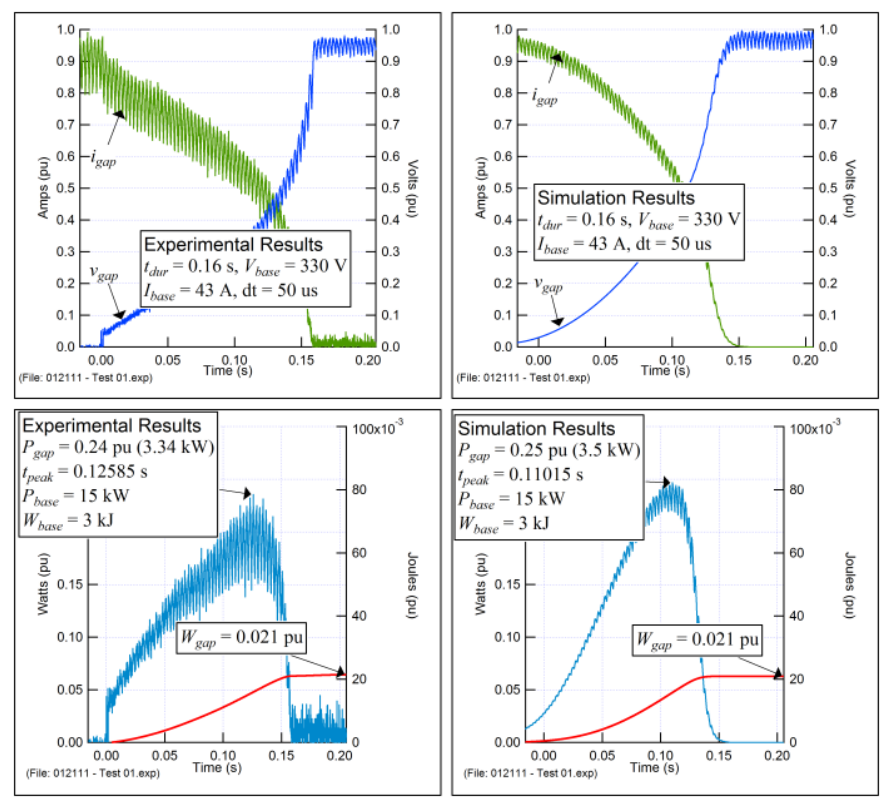

Fig. 12. Case study 3: accelerated fault (top row: instantaneous voltage and current; bottom row: instantaneous power and cumulative energy).

In addition to validating the model via its time domain voltage and current waveforms, the model was validated via its power trend, peak power, and energy consumption.

If the arc energy is similar in both experimental and simulation results, it implies that the model is consistent with what occurs in practice. This validation is an important consideration for model accuracy. The simulation model presented instantaneous voltages, currents, power, and energy that are close to what is measureable experimentally, and is deemed suitable for electromagnetic simulations. 


\section{ACKNOWLEDGMENTS}

The authors thank the Office of Naval Research for their financial support, and Dr. Mark Flynn and Mr. Tim Beets (both of UT-CEM) for the design, construction, commissioning, and data acquisition of the series fault test apparatus.

\section{REFERENCES}

[1] S. Kwon, et al., "Current development and future plan for smart distribution grid in Korea," in CIRED Seminar: SmartGrids for Distribution, 2008, p. 141.

[2] U.S. Department of Energy Office of Electricity Delivery \& Energy Reliability Office of Electricity Delivery \& Energy Reliability, "Smart Grid Research \& Development: Multi-Year Program Plan," ed, 2010-2014.

[3] A. Ziani and H. Moulai, "Extinction properties of electric arcs in high voltage circuit breakers," J. Physics D: Applied Physics, vol. 42, pp. 1-6, 2009.

[4] F. Karetta and M. Lindmayer, "Simulation of the gas dynamic and electromagnetic processes in low voltage switching arcs," IEEE Trans. Comp. Packag. \& Mfg. Technology, vol. 21, pp. 96-103, 1998.

[5] R. F. Ammerman, et al., "DC arc models and incident energy calculations," in IAS 56th Annual Petroleum and Chemical Industry Conference, Anaheim, CA, 2009.

[6] V. V. Terzija and H. J. Koglin, "On the modeling of long arc in still air and arc resistance calculation," IEEE Trans. Power Delivery, vol. 19, pp. 1012-1017, Jul 2004.

[7] J. Andrea, et al., "A new DC and AC arc fault electrical model," in 56th IEEE Holm Conference on Electrical Contacts, Charleston, SC, 2010, pp. 1-6.

[8] A. Parizad, et al., "Optimization of arc models parameters using genetic algorithm," in Int'l. Conf. Elec. Power \& Energy Conv. Sys., Ottawa, Canada, 2009, pp. 1-7.

[9] J. D. Herbst, et al., "Flexible test bed for MVDC and HFAC electric ship power system architectures for navy ships," in Electric Ship Technologies Symposium, Alexandria, VA, 2011.

[10] R. Hebner, et al., "Intelligent microgrid demonstrator," in ASNE Electric Machines Technology Symposium, Philadelphia, PA, 2010.

[11] R. Hebner, J. Herbst, and A. Gattozzi, "Large scale simulations of a ship power system with energy storage and multiple directed energy loads," in Grand Challenges in Modeling \& Simulation (GCMS 2010), Ottawa, Canada, 2010, pp. 430-435.

[12] F. M. Uriarte and V. Centeno, "High-Impedance Fault Detection and Localization in Distribution Feeders with Microprocessor Based Devices," in the North American Power Symposium (NAPS), Iowa State University, 2005, pp. 1-6.

[13] D. R. Doan, "Arc flash caculations for exposures to DC systems," IEEE Trans. Industry Applications, vol. 46, pp. 2299-2302, Nov 2010.

[14] V. V. Terzija, et. al., "EMTP simulation and spectral domain features of a long arc in free air," in 18th Int'l Conf. Electric Distribution, 2005, pp. 1-4.

[15] M. B. Djuric and V. V. Terzija, "A new approach to the arcing fault detection for fast autoreclosure in transmission systems," IEEE Trans. Power Systems, vol. 10, pp. 1793-1798, 1995.

[16] J. Trelles, et al., "Multiscale finite lement modeling of arc ynamics in a DC plasma torch," Plasma Chem. Plasma Process, vol. 26, pp. 557-575, 2006

[17] A. Pratt, et al., "Evaluation of 400V DC distribution in telco and data centers to improve energy efficiency," in 29th International Telecommunications Energy Conference (INTELEC), 20077, pp. 32-39.

[18] D. Nilsson and A. Sannino, "Efficiency analysis of low- and medium- voltage DC distribution systems," in IEEE Engineering Society General Meeting, 2004, pp. 2315-2321.

\section{BIOGRAPHIES}

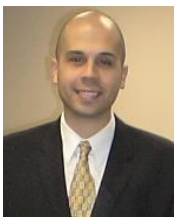

Fabian Marcel Uriarte, PhD is a Research Associate at the Center for Electromechanics at the University of Texas at Austin. He obtained his BS and MS from Virginia Tech, and $\mathrm{PhD}$ from Texas A\&M University-all in electrical engineering. His research interests are parallel simulation and modeling of power systems. He is currently involved in dc arc modeling and in the simulation of smart grids including residences, photovoltaic arrays, electric vehicles, and energy storage.

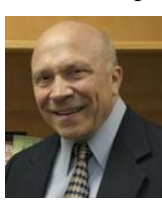

Angelo L. Gattozzi, PhD is a Research Associate at the Center for Electromechanics at the University of Texas at Austin where he has been working on power modules for the electric gun program, resonant converters for high speed motors/generators with flywheel energy storage, energy harvesting from sea waves, and simulations for the electric system of the DDG51 class Navy destroyer. Prior to joining the University of Texas, he was responsible, at the Lincoln Electric Company in Cleveland, Ohio, for the development of a complete new line of induction motors, achieving the highest efficiency levels in the industry.

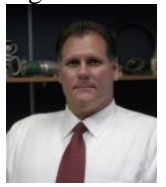

John D. Herbst is currently the Principal Investigator for research programs involving high power rotating electric machines and power converters at the Center for Electromechanics at the University of Texas at Austin. Prior to his current position, Mr. Herbst served as Principal Investigator for the ONR Megawatt Power Module for Ship Service program, an effort to explore high speed generators and energy storage flywheels to reduce fuel consumption on the DDG51 class of Navy warships. He was also Co-Principal for the Advanced Locomotive Propulsion System (ALPS) project, a $\$ 30 \mathrm{M}$ effort to demonstrate an advanced hybrid electric propulsion system for high speed passenger locomotives.

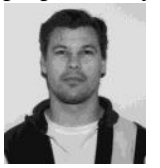

Hunter B. Estes is a graduate student in electrical and computer engineering and Research Assistant at the Center for Electromechanics at the University of Texas at Austin. He obtained his BS in chemical engineering, his MS in electrical engineering, and is currently pursuing a $\mathrm{PhD}$. His research group is Energy Systems, under the guidance of Dr. Alexis Kwasinski and Dr. Robert E. Hebner. His interests are in solar generation, as well as smart grid energy management.

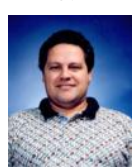

Thomas J. Hotz is a Research Engineering Associate V at the Center for Electromechanics at the University of Texas at Austin. He obtained his BS in electrical engineering at the University of Texas at Austin. His research areas include control and data acquisition systems for numerous experiments including microgrid, electromagnetic vibrators, using algae as a bio-fuel, and various power systems.

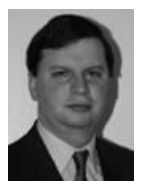

Alexis Kwasinski, PhD is an Assistant Professor in the Department of Electrical and Computer Engineering at the University of Texas at Austin. He obtained his MS and PhD in electrical engineering from the University of Illinois at UrbanaChampaign. His past industry experience includes working for Telefónica of Argentina and for Lucent Technologies Power Systems. His research interests include power electronics, distributed generation, renewable and alternative energy, smart grids, and analysis of the impact of natural disasters on critical power infrastructure. In 2005, he was awarded the INTELEC Fellowship and in 2007 he received the best technical paper award at INTELEC. In 2009 he received an NSF CAREER award. Dr. Kwasinski is an Associate Editor for the IEEE Transactions on Energy Conversion.

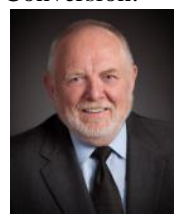

Robert E. Hebner, PhD (S'70-M'71-SM'83-F'93) is Director of the Center for Electromechanics at the University of Texas at Austin. The Center develops technology, primarily for novel motors, generators, and suspension components, and teams with companies to get the technology into the market. Previously, Dr. Hebner was the acting Director of the U.S. National Institute of Standards and Technology (NIST). In addition, he has directed NIST's Electronic and Electrical Engineering Laboratory, a laboratory with a staff of more than 250. 